\title{
Algunas consideraciones sobre el origen de los términos hormigón y concreto
}

\author{
M. ${ }^{a}$ TERESA SOLESIO DE LA PRESA
} Leda. en Filosofia y Letras (Historias) - IETCC/CSIC

\section{$R E S U M E N$}

En este trabajo se estudia, en primer lugar, una cierta manera de construir que practicaban los antiguos habitantes de la Península Ibérica, y que vieron los romanos a su llegada. Esta forma de construir consistía en hacer paredes de tierra llamadas "formaceos" y que son la base de un rudimentario hormigonado.

A través de los tiempos esta palabra "formaceos" fue derivando hasta llegar a "formicare", como sinónimo de "formare"; a su vez este "formicare", por diferentes motivos fonéticos, nos da el término "hormigar" $y$ añadiendo el sufijo $O N$, propio del aumentativo, tenemos la palabra hormigón.

Por otro lado, en este trabajo se estudia, asimismo, el término "CONCRETO", palabra cada vez más usada en países de habla hispana para designar el término hormigón, por influencia de los paises de habla sajona.

Nunca la palabra "concreto" ha servido para designar un material de construcción, y sólo es a partir del siglo XIX cuando, seguramente, se usa para este fin, con objeto de dar a este material un matiz más cientifico.

Por este motivo, se pretende en este trabajo aclarar que, teniendo nuestro idioma una acepción como la palabra hormigon - referida a material de construccióny que figura en nuestros Diccionarios desde 1516, no debemos aceptar la palabra "concreto" $y$, sobre todo, debemos intentar que su uso no se generalice en paises de habla hispana.

\section{$S U M M A R Y$}

In this work there is studied, in the first place a certain manner of constructing which was practiced by the ancient inhabitants of the Iberian Peninsula, and which was seen by the Romans on their arrival. This form of constructing consisted in making walls of earth called "formaceos", and which are the basis of a rudimentary concrete.

Through the ages this word "formaceos" was modified to "formicare", as a synonim of "formare"; in turn this word "formicare", for different phonetic reasons, gives us the term "hormigar" (to make concrete), and by adding the augmentative prefix ON (in the Spanish language) we have the term "hormigón" (concrete).

On the other hand, in this work there is likewise studied the term "CONCRET", (concrete) a word increasingly used in Spanish speaking countries, through the influence of the English speaking countries.

The word "concreto" (concrete) has neve been used to designate a building material, and it is only from the 19th century when, surely, it is used for this object. in order to give this material a more scientific nuance.

For this reason, this work, with the object of clarifying the matter, states that as our language accepts the word "hormigón" (concrete) - referring to building material-and which is included in our dictionaries since the year 1516, we should not accept the word "Concreto" (concrete) and, above all, we should try to prevent its use being generalized in the Spanish speaking countries. 


\section{ANTECENDENTES DEL TERMINO HORMIGON}

El estudio de la palabra HORMIGON tiene ciertas dificultades, desde el punto de vista morfológico. Como se verá, en el presente trabajo, se parte de dos hipótesis más frecuentes y, a su vez, se ha estudiado una tercera, apoyándose en la Gramática Histórica Española del Profesor Menéndez Pidal.

Asimismo, se hace un bosquejo histórico del término hormigón, basándose en la recopilación bibliográfica encontrada y que hace mención al tema.

\subsection{Origen romano}

Cuando los romanos llegaron a España y a Africa vieron cómo, en estos paises, se acostumbraba a construir muros, por medio de una armadura de madera, apisonando piedras y arcilla dentro de una especie de cajones; dichos muros, una vez retirados los cajones, quedaban compactos, como si hubieran sido hechos con el mejor cemento.

Plinio a este propósito dice: (Plinio. Naturalis Historia XXXV, 169) (1).

"Cómo que no existen en Africa ni en España paredes de tierra a las que llaman "formaceos", puesto que una vez dispuestas las planchas de madera a modo de moldes llenos se endurecen con el tiempo y resisten a las lluvias, a los vientos, al fuego y son más resistentes que cualquier clase de cemento. Pues bien, fijate en los puestos de vigilancia de Hanibal, en España, y en las torres hechas de ticrra colocadas en las cimas de los montes".

Asimismo se encontraron los romanos con casas, de gran altura, construidas con una solidez extraordinaria a causa de la compacidad conseguida por la unión de piedras y mortero.

Varro en (De re rústica. I, 14, 4) (2) escribe:

"El cuarto cercado fabril (hecho de artesanía) es muy característico: paredes de adobes... compuestas de tierra y guijarros colocados en moldes"...

De estos PARIES FORMACEUS, que habla Plinio, deriva la palabra HORMAZO con el significado de "molde", "horma", "forma". San Isidoro, asimismo, comenta esta palabra, pero ya abreviada en FORMACIUS, como vocablo propio de España y Africa usado en la antigüedad.

\section{ETIMOLOGIA}

Sobre la palabra HORMIGON se dan diversas hipótesis etimológicas.

\subsection{Primera hipótesis}

HORMIGON deriva de FORMICARE, contruir con tapial, y esto no es hipotético puesto que a principios del siglo XI lo usó el francés Dudon, si bien como mero sinónimo de FORMARE

(1) Quid". non in Africa Hispaniaque e terra parietes, quos appellant formaceos, quoniam in forma circumdatis II utrimque tabulis inferciuntur verius quam struuntur, aevis durant, incorrupti imbribus, ventis, ignibus, omnique caemento firmiores? spectat etiam nunc speculas Hannibalis Hispania terrenasque turres, iugis montium inpósitas.

(2) Quartum fabrile saepimentum est novissimum, maceria.. quod ex terra et capillis compositis in formis...

70 

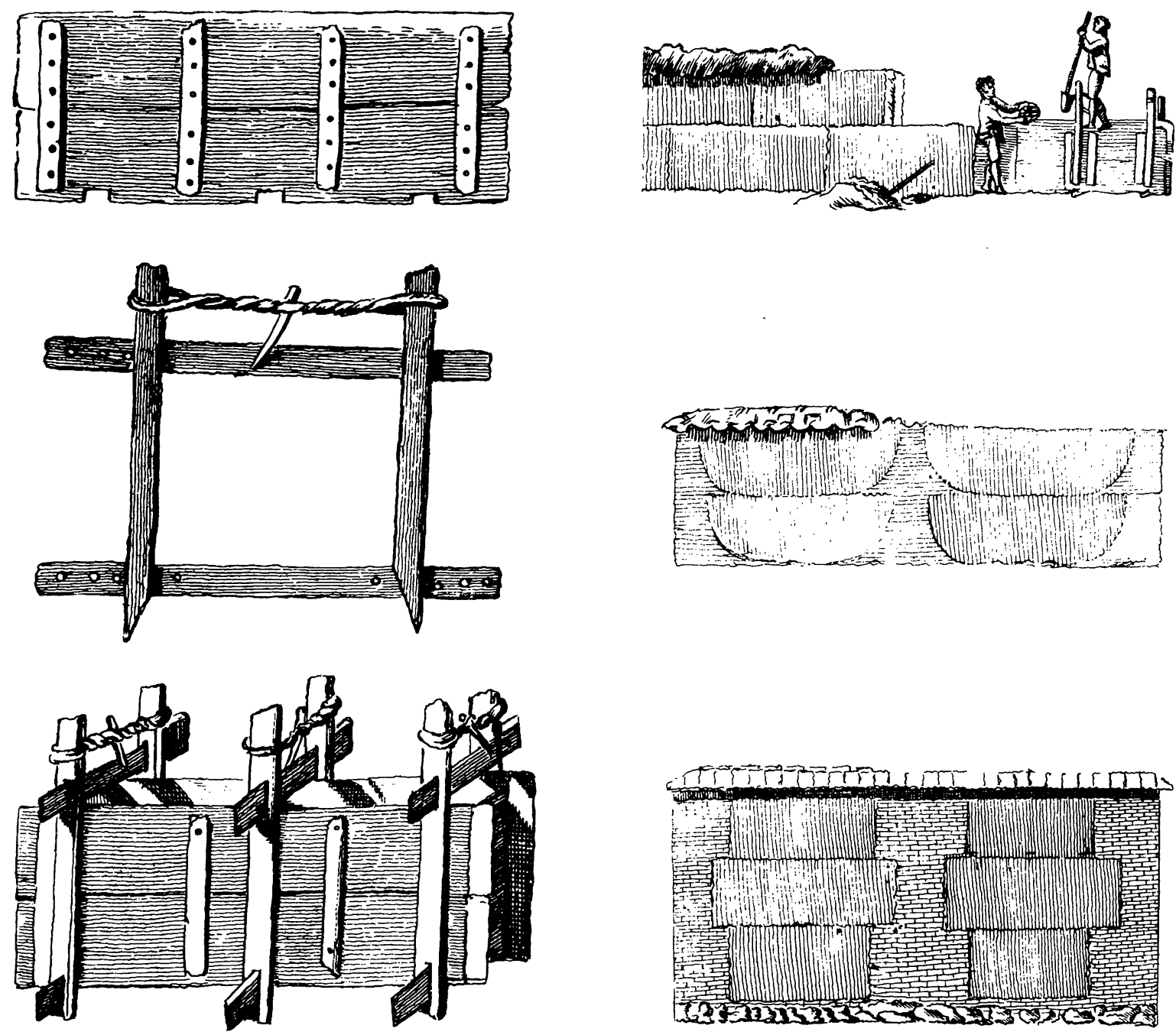

Villanueva, Juan de. Arte de Albañilería. Madrid 1827.

(confrontar DU CANGE Glossarium Mundiae et Infimae Latinitatis... Niort, L. Favre, 1883-1887. 10 vols).

Covarrubias (Tesoro de la Lengua Castellana; impresión de 1661; con las adiciones de Benito Noydens. 1674; Barcelona 1943) sugiere que la palabra HORMIGON viene de "FORMA" y que se dijo asi por hacerse entre dos tapiales, que son forma de pared y comenta la cita de Plinio, anteriormente mencionada.

Por otra parte, en el Medieval latin word - list from British and Irish Sources de J. H. BAXTER y C. JOHNSON (London, Oxford University Press, 1947), se registra la voz "FORMIFICO" en el sentido, igualmente, de dar forma.

En el Vocabulario del Romance, en latin, de Elio Antonio Nebrija (Sevilla 1516) se cita "pared de hormigón", con la significación de "crusta calcaria".

El Padre Juan de Mariana, en su Historia de España, libro 17, cap. 13 (1), comenta la palabra HORMA para significar la pared o muralla de piedra sobrepuesta (paries ex solis

(1) "a la hora le hizo cerca de una horma, pared de piedra seca"... 
lapidibus) (confrontar Diccionario de Autoridades. Diccionario de la Lengua Castellana, Madrid, Real Academia Española, 1726-1739, 6 vols. Edición Facsimil, Madrid, Gredos, 1969, 3 vols.).

Esta primitiva palabra FORMA, aplicada propiamente al molde de la tapia, con el tiempo y el uso pasa a designar, también, la tapia misma.

De igual manera, en vasco, existen las palabras BORMA y ORMA con el sentido de "pared", y en gascón ARROUME significa "muralla de piedras superpuestas" (ARROUMA "former une telle muraille"). (Confrontar COROMINAS. Diccionario Crítico Etimológico de la Lengua Castellana. Editorial Gredos, Madrid, 1954, 4 vols.).

El Diccionario de la Real Academia ha recogido S. V. HORMA en su 2." acepción: "pared de piedra".

Partiendo de todo lo expuesto tenemos el siguiente esquema:

\begin{tabular}{|l|l|l|}
\hline & \multicolumn{1}{|c|}{ "MOLDE” } & \multicolumn{1}{c|}{ "PARED" } \\
\hline LATIN & FORMA & $\begin{array}{l}\text { PARIES FORMACEUS (Plinio) } \\
\text { FORMACIUS (S. Isidoro) } \\
\text { HORMAZO español }\end{array}$ \\
\hline ESPAÑOL & FORMA/HORMA & HORMA (P. Mariana) \\
\hline LATIN | MEDIEVAL & FORMICARE (sinónimo FORMARE) & HORMIGON \\
\hline
\end{tabular}

Por todo esto, así como está atestiguada la palabra FORMA = molde y PARIES FORMACEUS, que es propiamente la pared hecha con este molde y que se conoce en español con la palabra HORMAZO; de la misma manera tenemos la palabra HORMIGON, que viene a sustituir al PARIES FORMACEUS, o sea al HORMAZO y que está relacionada con el FORMICARE como sinónimo de FORMARE.

Esta relación se puede explicar con la siguiente hipótesis:

\subsection{Segunda hipótesis}

\section{FORMICARE}

- La sustitución de la $\mathrm{F}$ inicial por $\mathrm{H}$ y subsiguiente pérdida es un fenómeno primitivo debido a la influencia ibérica de los dialectos vecinos al vasco (confrontar Menéndez Pidal R. Manual de Gramática Histórica Española, VII edición, Madrid, 1944, párrafo 38).

\section{HORMICARE}

- Las consonantes oclusivas sordas latinas $P$ - $T$ - K $(C$ sonido $K)$, en posición intervocálica, se sonorizan en B - D - G (confrontar Menéndez Pidal, obra citada, párrafo 40).

\section{HORMIGARE}

- La E final se pierde siempre después de las consonantes $\mathrm{T}-\mathrm{D}-\mathrm{N}-\mathrm{L}-\mathrm{R}-\mathrm{S}$ y $\mathrm{C}$ (confrontar Menéndez Pidal, obra citada, párrafo 28). 


\section{HORMIGAR}

- El AR es terminación propia de infinitivo, con lo cual tendríamos HORMIG y si añadimos un sufijo "ON", propio de aumentativo, nos queda HORMIGON. Este sufijo aumentativo "ON", no es dudoso pensar, fuese debido a la resistencia y la duración del propio material, e incluso por usarse, frecuentemente, en grandes masas.

\subsection{Tercera hipótesis}

Otra hipótesis relaciona HORMIGON con HORMIGOS "gachas", comida hecha con trigo o maiz quebrantados o pan desmenuzado, cocidos con agua o leche (1).

Según Corominas (Diccionario Crítico Etimológico de la lengua Castellana), obra citada "de origen incierto, quizá derivado de HORMIGA por comparación de los granos de trigo que quedan en el arnerillo o en las gachas con las hormiguillas que bullen en el hormiguero".

Para apoyar esta hipótesis que relaciona HORMIGON (mezcla compuesta de piedras menudas y mortero de cal y arena) con HORMIGOS (gachas, postre de avellanas machacadas) su relación se describe en el siguiente esquema:

\begin{tabular}{|l|l|}
\hline $\begin{array}{l}\text { HORMIGOS } \\
\text { postre de avellanas macháadas }\end{array}$ & $\begin{array}{l}\text { HORMIGON } \\
\text { mezcla compuesta de piedras menudas y de cal y arena }\end{array}$ \\
\hline $\begin{array}{l}\text { NUEGADO } \\
\text { (postre de avellanas machacadas) }\end{array}$ & $\begin{array}{l}\text { NUEGADO } \\
\text { (hormigón) }\end{array}$ \\
\hline $\begin{array}{l}\text { PINYO } \\
\text { (piñon) }\end{array}$ & $\begin{array}{l}\text { PINYOLENC } \\
\text { (mineral de conglomerados) }\end{array}$ \\
\hline
\end{tabular}

\section{ANTECEDENTES DEL TERMINO "CONCRETO"}

El estudio de la palabra "CONCRETO", en el sentido de material de construcción, presenta serias dificultades, por no ser palabra castellana y ser su denominación, bajo esta acepción, relativamente reciente. Sin embargo, esto no ha sido óbice para que dicho anglicismo haya tenido una gran difusión, pasando incluso a formar parte, y de manera muy arraigada, en paises de habla hispana.

\subsection{Origen latino}

La palabra "CONCRETO" según el Thesaurus Lingua Latinae, Tomo IV. Leipzig 1906-1909, significa "espeso, condensado, compacto, no fluido", es decir, siempre refiriéndose a estados de la materia y nunca con sentido de material de construcción, también en sentido filosófico y matemático.

CONCRETUM en su forma de sustantivo, y según Claudius Donatus (siglo IV), y en su comentario a la Eneida de Virgilio, dice "lo que de muchas cosas pequeñas se hace una sola masa". Sin embargo este sustantivo ha sido siempre muy poco usado.

(1) Asimismo, "partes más gruesas que quedan en el arnerillo al cribar la sémola o trigo quebrantado". "plato de reposteria hecho con almendras o avellanas tostadas y machacadas con micl". 


\subsection{Origen inglés}

En la lengua inglesa "CONCRETE" procede del latin y su uso era ya corriente en $1400 \mathrm{ME}$ (Inglés Medio), pero siempre con el sentido de espeso, condensado.

Según The Oxford English Dictionary, vol. II. Oxford Clarendon Press, 1933. CONCRETE, en su tercera acepción específica, se define como "una composición de piedras menudas, arena, grava, guijarros, etc.", que forman una masa con el cemento. $Y$ en esta forma de sustantivo y haciendo referencia a material de construcción, aparece por primera vez en 1834: Revista Lond Archit. Mag. I. 35, ejecución de una cimentación artificial de "concreto" (que se ha hecho recientemente en muchos lugares). En 1836, G. Godwing en Trans. Inst. Brit. Archit. 12, afirma que el término genérico "concreto" tal vez sólo pueda datar del periodo en que su uso se generalizó y se hizo frecuente, probablemente, no hace más de 15 ó 20 años. En 1858, Glenny Gard en Everey-day BK 25/1 habla de pavimentos de ladrillo, teja, piedra o "concreto" y, en 1881, Darwin Form. Veg. Mould 181, escribe sobre la unión de un suelo de "concreto", con los muros. En este mismo diccionario "CONCRETO", en su forma transitiva y en la cuarta acepción dice: tratar con "concreto", usar o aplicar "concreto" en la construcción de edificios; aparece por primera vez en 1875 en "Building New 2 Apr. 390" en dos artículos: vaciado del "concreto" (hormigonado). En 1882 en "Daily New, 15 Sept. 6 5" habla del vaciado del "concreto" en cimentaciones (hormigonado de cimentaciones). En 1885 Du Cane, en Punishm $\&$ Prevent. Crime 180, publica sobre: hinca de pilotes y vaciado del "concreto" para cimentaciones (hinca de pilotes y hormigonado de cimentaciones).

Shipley, J. T. (Dictionary of Word Origins. New York: Philosophical Library, 1945, pág. 93) dice: la mezcla de guijarros, grava, arena, etc. con cal o cemento... se llama "CONCRETO", porque se forma de una sola masa. Para Skeat, W.W. (A Concise Etymological Dictionary of the English Languaje. New York: Capricorn Books, 1963, pag. 105) es: formar una masa, del latín concret-us, participio pasivo de concrescere, crecer junto.

Como se ve, las primeras menciones sobre la palabra "concreto" surgen en Inglaterra, en época muy tardia, ya en pleno siglo XIX. La Enciclopedia Británica señala cómo la palabra "CONCRETO", sin término calificador, se usó, poco más o menos, a partir de 1900, para designar, de forma abreviada, al Portland Cement Concrete.

\subsection{Origen castellano}

En nuestro idioma la palabra CONCRETO es un derivado de crecer, del latín CRESCERE, en cl sentido de espeso, condensado, compacto, crecer por aglomeración, espesarse, endurecer.

En este sentido figura ya (1499) en Juan de Mena, en su obra Trescientas con la Glosa de Fernán Núñez. Asimismo, derivado de crecer es concretar y concreción del latín concretio-onis, agregación, materia y derivado del mismo verbo concrecionar y concrescencia. Terreros y Pando (Diccionario Castellano con las voces de Ciencias y Artes y sus correspondientes en las tres lenguas francesa, latina e italiana. Madrid 1786-93, 4 vol.) cita concretar, pero siempre con referencia a la materia; en cambio falta esta palabra en el Diccionario de Autoridades: Diccionario de la Lengua Castellana, Madrid, Real Academia Española 1726-1739, 6 vols. Edición Facsimil, Madrid 1963, 3 vols. El Diccionario de la Real Academia de la Lengua, Madrid 1956, en su 18 edición cita, por primera vez, la palabra "CONCRETO" con el significado de hormigón armado, pero referido a América. El Diccionario de Anglicismos de Ricardo J. Alfaro, Madrid, Gredos, 2." edición aumentada en 1970 registra la palabra "CONCRETO" como un anglicismo del sustantivo hormigón. 


\subsection{Americanismo}

La palabra CONCRETO pasó a Hispanoamérica en época muy reciente, y ésta es la causa de que en el Diccionario de Americanismos de Augusto Malaret, 2. ${ }^{a}$ edición, Puerto Rico 1931, no figure "CONCRETO" como material de construcción; en cambio en el Diccionario General de Americanismos de Francisco Santamaría, edición Pedro Robredo, México 1942, ya figura este término, que con excepción de Argentina y Chile se ha hecho fuerte en todos los países de habla hispana. Como dato significativo el colombiano Cuervo apunta que sería más correcto, refiriéndose a Colombia, usar la palabra "gorgón" en lugar de hormigón, pero nunca "concreto".

\section{CONCLUSIONES}

A partir de estos antecedentes podemos establecer las siguientes premisas:

a) Concretus, palabra latina, tiene el sentido de espeso, condensado, compacto, no fluido y siempre referido a estados de la materia.

b) Asimismo "concreto", palabra castellana derivada del latín, también tiene el mismo sentido y jamás se ha usado como palabra para designar un material de construcción.

c) Igual pasa con concrete, palabra inglesa, y es a partir de 1834 cuando surge para designar un material de construcción. Es posible, que algún cultista inglés, basándose en Claudius Donatus y queriendo dar al nuevo material un matiz cientifico, se acordase del sustantivo concretus.

d) Por influencia inglesa pasa a hispanoamérica en fecha muy reciente.

e) Aunque en paises de habla hispana se haya generalizado la palabra "concreto", es un anglicismo que no está admitido por la Real Academia y, por lo tanto, esta palabra no debe ser usada.

f) Teniendo nuestro idioma una acepción como hormigón - con base histórica - anterior a la venida de los Romanos a España, referido a material de construcción y que figura en nuestros Diccionarios y Autoridades de la Lengua desde 1516, no debemos por ningún motivo emplear otro término y menos cuando éste no es correcto.

g) Por medio de este trabajo se pretende reivindicar la palabra HORMIGON en todo lugar, pero muy especialmente en paises de habla hispana. 